\title{
Interfacing between regional and global, electron density height profile
}

\author{
Bruno Zolesi $\left({ }^{1}\right)$ and Ljiljana R. Cander $\left({ }^{2}\right)$ \\ ( $\left.{ }^{1}\right)$ Istituto Nazionale di Geofisica, Roma, Italy \\ $\left.{ }^{2}\right)$ Rutherford Appleton Laboratory, Chilton, Didcot, Oxon, U.K.
}

\begin{abstract}
A first analysis of the possibility of interfacing regional and global electron density height profile models, over a buffer zone, was performed considering the results obtained using different models such as IRI 90 (Bilitza, 1990), DGR (Di Giovanni et al., 1992) and RAL MPQ (Dick and Bradley, 1992). The differences $\Delta N \cdot \mathrm{cm}^{-3}$ of electron density as a function of altitude between different models were calculated using monthly median experimental data measured at Rome station and values predicted by URSI, SIRM (Zolesi et al., 1993) and EOF (Singer and Dvinskikh, 1991) mapping models for the same point. Composite ionograms are used to roughly evaluate the performances of the three models considered.
\end{abstract}

Key words ionosphere - ionospheric modelling

\section{Introduction}

PRIME (COST 238) a Project of the European Union (Bradley, 1992) is concerned with developing ionospheric maps and models within Europe so that in any final user software package a smooth interface to international adopted global maps and models must be provided. Numerical and analytical smoothing methods for interfacing $f_{0} F_{2}$ and $M 3000 F_{2}$ between regional and global maps have been proposed by Harrison (1992) and Leitinger (1993) to be applied on the intermediate buffer zone. Outside the PRIME area the standard density height profile is the IRI model having as input the ionospheric data coming from global models and formulas such as CCIR, URSI, etc.

Mailing address: Dr. Bruno Zolesi, Istituto Nazionale di Geofisica, Via di Vigna Murata 605, 00143 Roma, Italy; e-mail: ZOLESI@MARTE.INGRM.IT
If inside the PRIME area we still adopt the IRI model, having as input the data coming from the regional models, the problem of interfacing in the buffer zone is only a problem of smoothing the ionospheric characteristics solved by Harrison and Leitinger. If we adopt instead, other models such as DGR or RAL MQP we have to consider the eventual discontinuities of electron density at fixed heights as well as errors introduced by the different regional models.

\section{Data analysis}

Discontinuities of electron density between different models in a fixed point and altitude are analyzed in three steps, considering their differences $\Delta N \cdot \mathrm{cm}^{-3}$ as a function of height. In the first step, shows the behaviour of $\Delta N \mathrm{~cm}^{-3} v s$. height calculated comparing the electron density profile obtained using IRI 90 (here assumed as the global model) with the electron density profiles obtained using the 
other two models DGR and RAL MQP (assumed as regional models). The input data applied are the median values measured at Rome station $\left(41.8^{\circ} \mathrm{N}, 12.5^{\circ} \mathrm{E}\right)$ during low solar activity 1986 for January and June at 00.00 and $12.00 \mathrm{~h} \mathrm{LT}$ (table I and fig. 1).

A first analysis of the behaviour of $\Delta N$ in 1990 , a period of very high solar activity, was made but not presented here. In fact, median values of $f_{0} F_{1}$ are calculated using very few data, so the graphs of this period not give a realistic result because the values are not representative of the month.

The second step is still a comparison of the differences of electron density vs. height between IRI with DGR, fig. 2 and IRI with RAL MQP, fig. 3. In this case, the input data are the predicted values in the same geographical point of Rome by the URSI model for IRI and by the two regional models proposed in PRIME, SIRM and EOF, both for DGR and RAL MQP.

The aim of the third step is to give a rough idea of the performances of the different models comparing their results with those coming from composite ionograms processed with the POLAN (Titheridge, 1985) procedure. Composite ionograms were obtained applying a simple tecnique to all the ionograms taken at Rome during the same months, years and local time as for the previous analysis (Zolesi et al., 1994).

Of course, composite ionograms is a discussed method so they should not be considered the real experimental median ionograms. Considering that many comparisons were made in the past between profiles coming from models and profiles coming from individual iono-

Table I. Median monthly values measured and predicted at Rome station $\left(41.8^{\circ} \mathrm{N}, 12.5^{\circ} \mathrm{E}\right)$ used as input data.

\begin{tabular}{|c|c|c|c|c|c|c|c|}
\hline & $H$ & $f_{0} F_{2}$ & $M(3000) F_{2}$ & $f_{0} F_{1}$ & $f_{0} E$ & $h m F_{2}$ & $R_{12}$ \\
\hline & \multicolumn{7}{|c|}{ Median values taken at Rome station $\left(41.8^{\circ} \mathrm{N}, 12.5^{\circ} \mathrm{E}\right)$} \\
\hline \multirow[t]{2}{*}{ January 1986} & 1200 & 5.8 & 3.67 & 3.70 & 2.85 & 207 & 14 \\
\hline & 0000 & 3.1 & 3.03 & & & 316 & 14 \\
\hline \multirow[t]{3}{*}{ June 1986} & 1200 & 5.2 & 3.01 & 4.37 & 3.25 & 235 & 14 \\
\hline & 0000 & 4.2 & 3.02 & & & 317 & 14 \\
\hline & \multicolumn{7}{|c|}{ Median values predicted at Rome station by SIRM } \\
\hline \multirow[t]{2}{*}{ January 1986} & 1200 & 6.2 & 3.61 & 3.18 & 2.69 & 207 & 14 \\
\hline & 0000 & 3.1 & 3.01 & & & 316 & 14 \\
\hline \multirow[t]{3}{*}{ June 1986} & 1200 & 5.6 & 3.02 & 3.58 & 3.35 & 235 & 14 \\
\hline & 0000 & 4.6 & 3.02 & & & 317 & 14 \\
\hline & \multicolumn{7}{|c|}{ Median values predicted at Rome station by EOF } \\
\hline \multirow[t]{2}{*}{ January 1986} & 1200 & 5.6 & 3.43 & 2.50 & 2.17 & 207 & 14 \\
\hline & 0000 & 2.5 & 2.93 & & & 316 & 14 \\
\hline \multirow[t]{2}{*}{ June 1986} & 1200 & 5.2 & 2.87 & 4.40 & 3.10 & 235 & 14 \\
\hline & 0000 & 4.7 & 2.97 & & & 317 & 14 \\
\hline
\end{tabular}



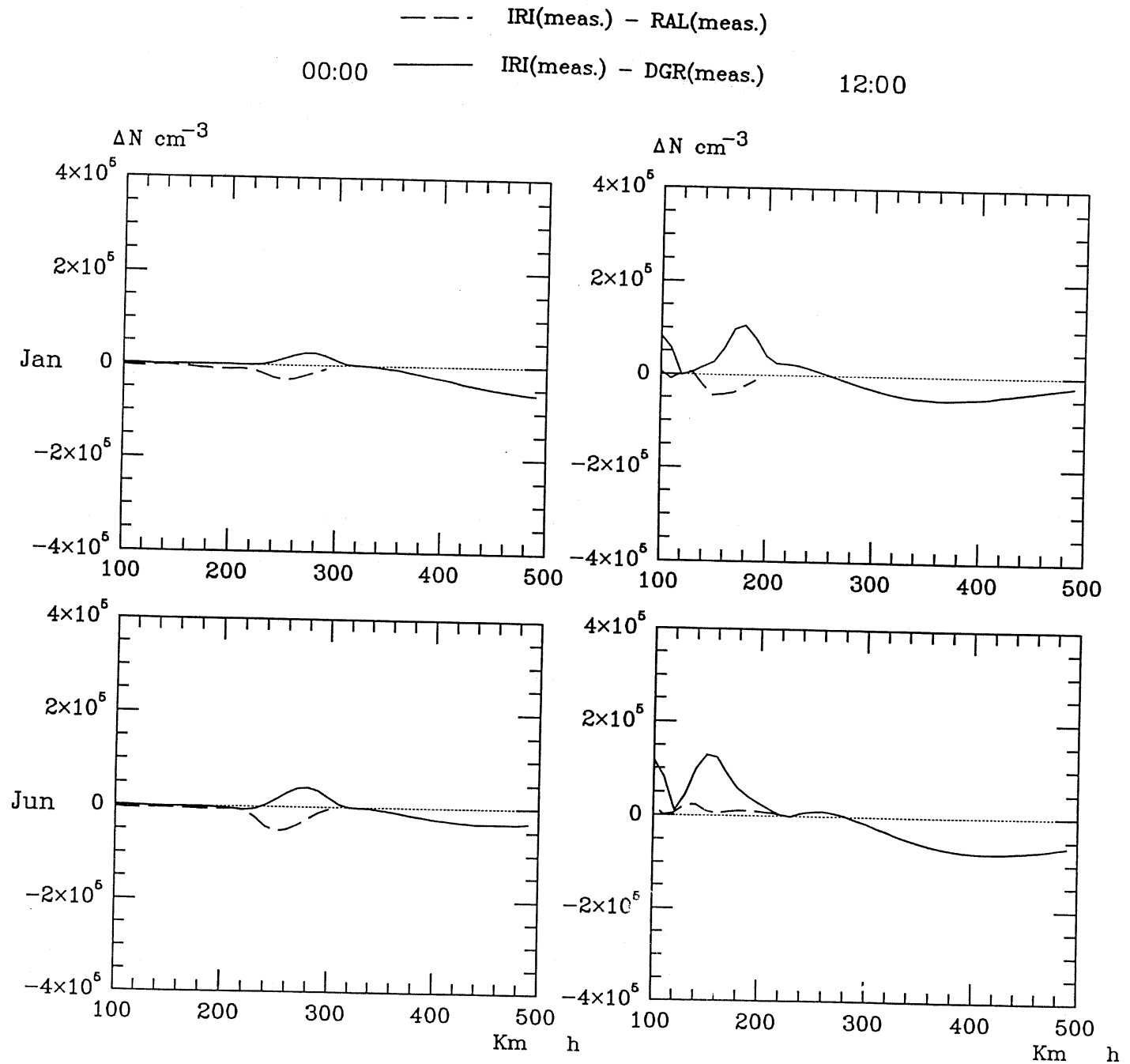

Fig. 1. Electron density differences $\Delta N \cdot \mathrm{cm}^{-3}$ vs. altitude $h$ between IRI and DGR models, continuous line, and between IRI and RAL models, dotted line, using median monthly values measured.

grams (Kecić et al., 1994; Singer et al., 1992), this analysis could give another contribution without claiming to be a testing procedure for electron density profile models.

Figure 4 shows for two different epochs the differences $\Delta N \cdot \mathrm{cm}^{-3}$ vs. height between the models IRI, DGR and RAL MQP and the electron density profiles coming from composite ionograms using as input data for the models the median measured values of table I.

A detailed analysis of the differences given by the three models, using predicted values of different mapping prediction methods as URSI, EOF and SIRM is reported in the Proceedings of PRIME workshops held in Eindhoven in 1994 (Zolesi et al., 1994). 


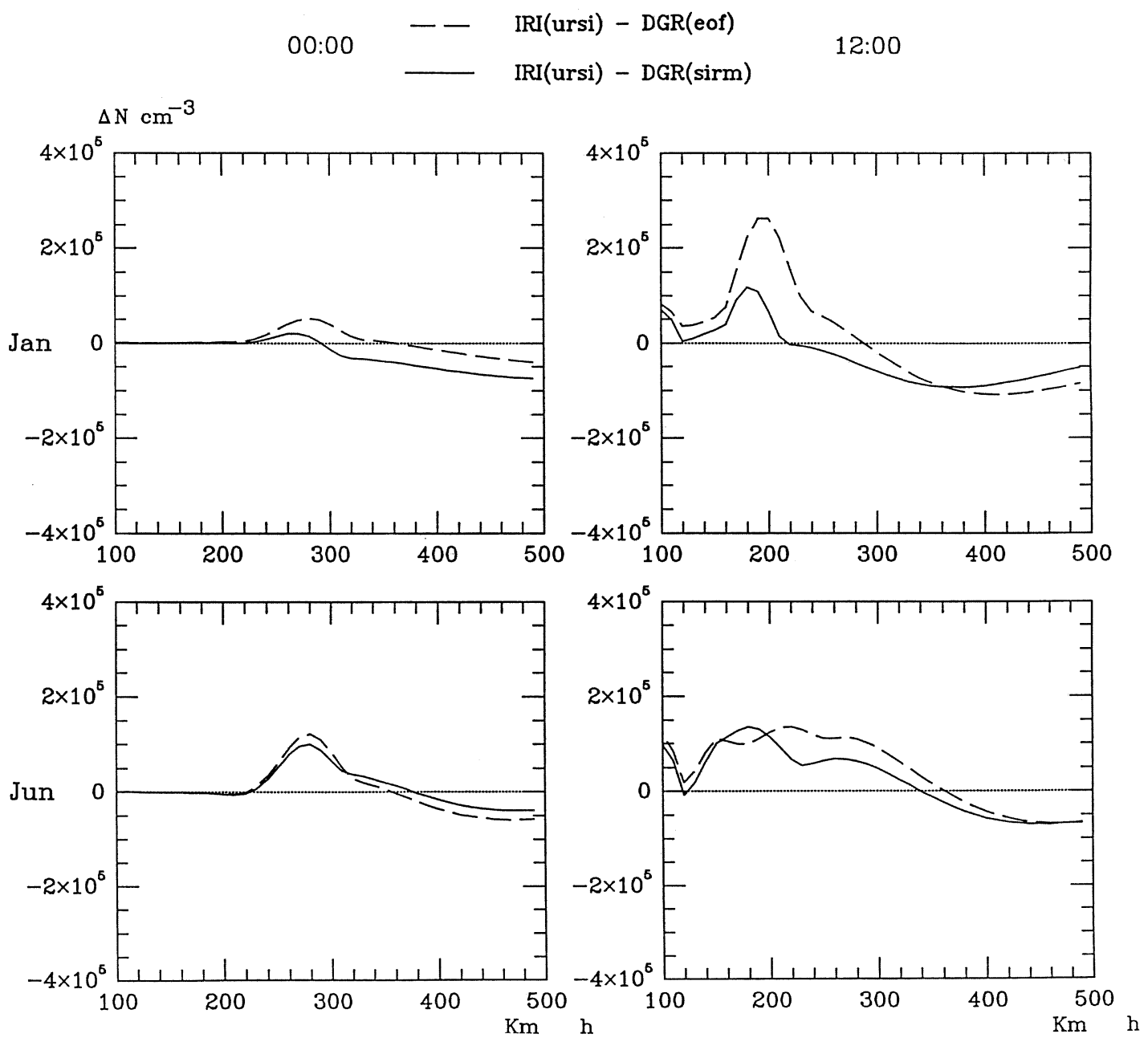

Fig. 2. Electron density differences $\Delta N \cdot \mathrm{cm}^{-3} v s$. altitude $h$ between IRI and DGR models using median monthly values predicted by URSI and SIRM models, continuous line, and by URSI and EOF models, dotted line.

\section{Discussion}

The models of electron density profile studied in this analysis, besides IRI, were DGR and RAL MQP because their software was available and because these two models were presented during PRIME Workshops for PRIME applications.

It is evident that a clear difference between the electron density profile given by IRI and that obtained by the other two models exists during the night hours and increases during noon, depending on different methods applied to calculate the electron density of the $F_{1}$ region. This trend is emphasized when the input data come from long term mapping models such as SIRM or EOF, introducing their errors and performances.

If a regional electron density is adopted in the PRIME area, a smoothing procedure should 


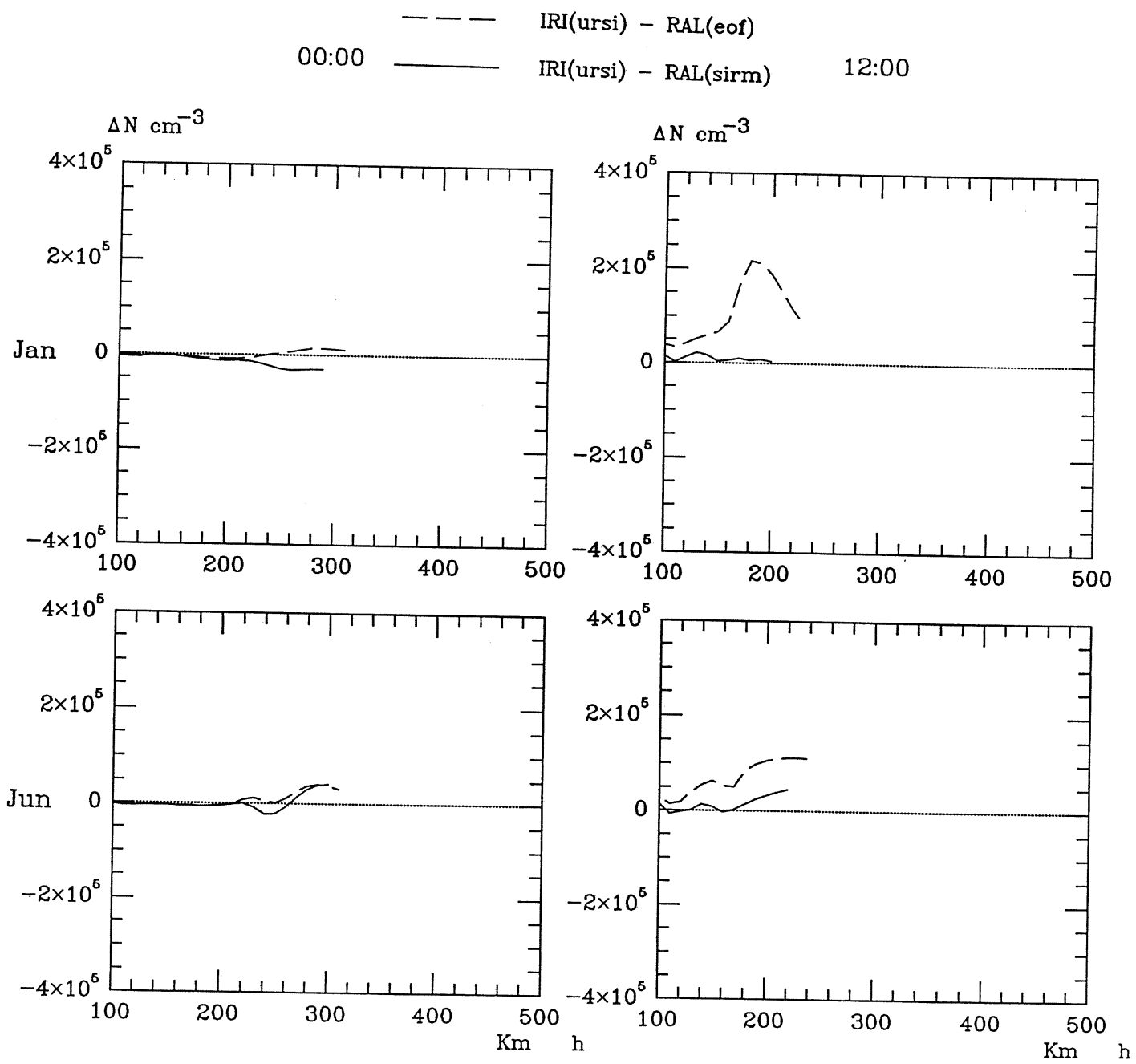

Fig. 3. Electron density differences $\Delta N \cdot \mathrm{cm}^{-3} v s$. altitude $h$ between IRI and RAL models using median monthly values predicted by URSI and SIRM models, continuous line, and by URSI and EOF models, dotted
line.

be applied in the buffer zone for every fixed height. The approach proposed by Harrison (1992) to switch a ionospheric characteristic from a regional to a global model is based on a simple weighting function:

$$
C \omega=\omega C_{r}+(1-\omega) C g
$$

where $C \omega$ is the ionospheric value predicted by the regional model, $C g$ the value predicted by the global model and

$$
\omega(x)=\frac{1}{2}\left[\cos \left(\frac{\pi}{b} x\right)+1\right]
$$

is a function of $b$ the width of the buffer zone, and $x$ the distance from the border of the regional zone. This procedure may be adopted 

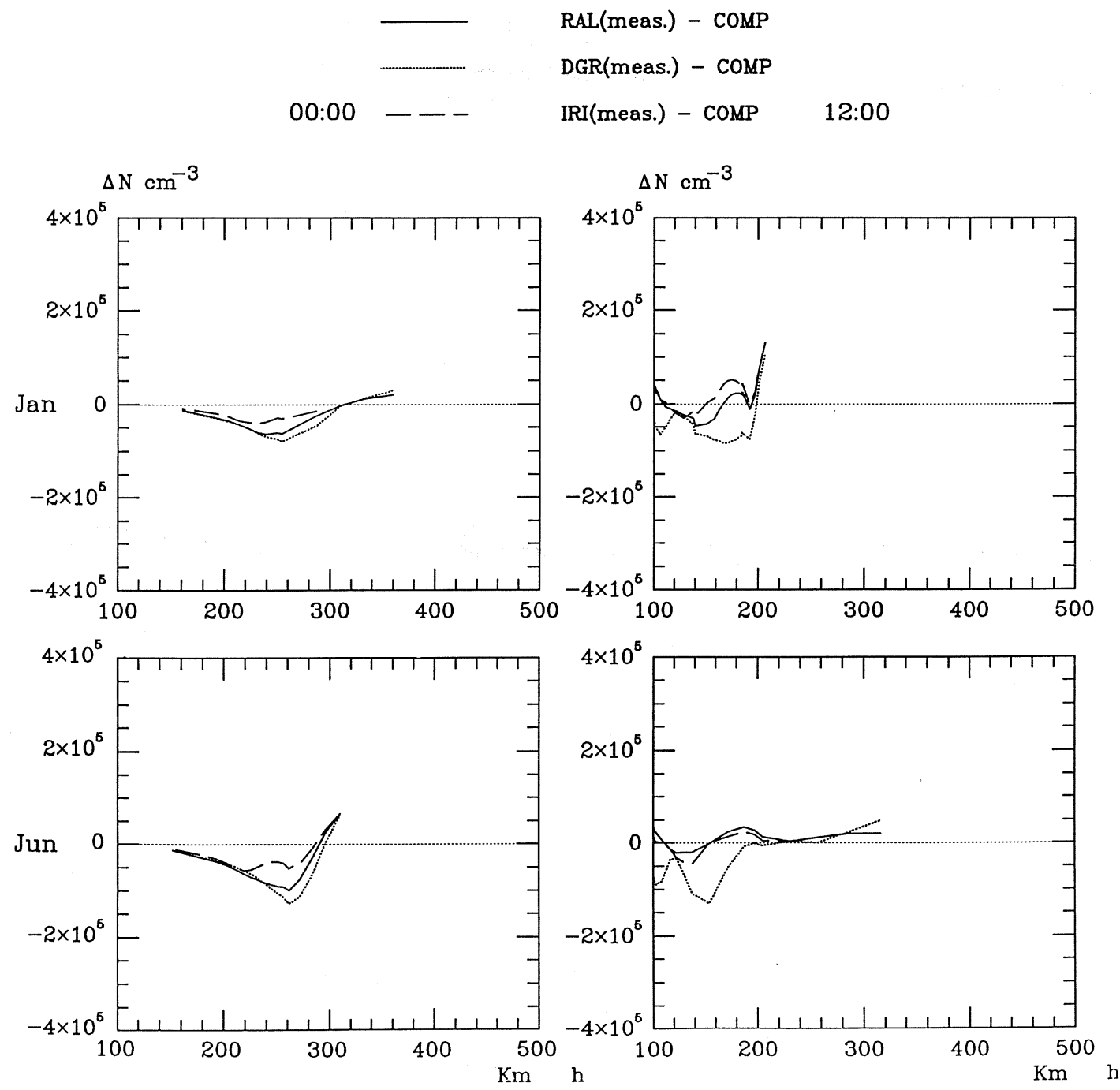

Fig. 4. Electron density differences $\Delta N \cdot \mathrm{cm}^{-3} v s$. altitude $h$ between IRI, DGR and RAL profiles, using median measured values, and profiles obtained by composite ionograms inverted with POLAN.

from all those users who only need reflection point data so a constant width buffer zone of 5 degrees may be used. Otherwise, to have a smooth transition between the regional and global model and to avoid strong changes in the required gradients by the same applications such as raytracing, the formula proposed by Leitinger (1993) should also be applied to in- terface electron densities at fixed heights:

$$
N(\phi, \lambda)=\frac{f(\phi, \lambda) \exp (\alpha d)+g(\phi, \lambda) \exp (-\alpha d)}{\exp (\alpha d)+\exp (-\alpha d)}
$$

where $N$ is the value in the buffer zone; $f$ and $g$ 
the values coming from the models to be joined; $\phi, \lambda$ (geographic) latitude and longitude; $\alpha$ the parameter which determined the width of the buffer zone and $d$ the distance from the centre, positive on the side of $f$, negative on the side of $g$.

\section{Conclusions}

The difference between IRI and RAL MQP is not so large because the latter model is an analytical approximation of the former. Results obtained comparing the profiles given by composite ionograms, even if this method is still under discussion, confirm the good performances of the three models also under median conditions. In any case, the decision to adopt a regional model for the electron density profile should depend not only on the possible performances of the different models, but also on the ease and practicability to interface mapping and electron density profile models. Great caution should be applied in the analysis of diurnal hours of high solar activity because of the rare occurrence of $f_{0} F_{1}$, with a frequent presence of $L$, not giving sufficient data to calculate suitable median values.

A meeting held in July 1994 at the Atmospheric Physics and Radiopropagation Laboratory of ICTP (International Center of Theoretical Physics) devoted to the discussion on the $F_{1}$ layer, pointed out the importance of this parameter for the electron density profile models. Considerations on global and regional models have been made concerning the seasonal occurrence and the latitudinal behaviour of this layer (Bilitza, 1995).

\section{Acknowledgements}

The authors thank P.A. Bradley for comments and suggestions and S. Radicella, M. Dick and W. Singer for the software programs of their models.

\section{REFERENCES}

BILITZA, D. (1990): International Reference Ionosphere 1990, NSSDC 90-92, Greenbelt, Ma. U.S.A.

BILITZA, D. (1995): The high latitude international reference ionosphere summary, Adv. Space Res., 16 (1), 1-4.

BRADLEY, P.A. (1992): PRIME (Prediction and Retrospective Ionospheric Modelling over Europe), First Annual Report (period March 1991 - May 1992), COST 238D(93)003.

Dick, M. and P.A. BRAdLey (1992): The RAL multiquasiparabolic model ionosphere, in Proceedings of the PRIME/URSI Joint Workshop on «Data Validation of Ionospheric Models and Maps», Roquetes (Tarragona) 4-8 May 1992, Memoria 16, 67-83.

Di Giovanni, G., S.M. Radicella and M.-L. ZHANG (1992): Further improvements of DGR ionospheric model and comparison with the IRI current model, COST238TD(92)021, May 1992.

HARRISON, S.M. (1992): Further studies of buffer zone smoothing between PRIME and CCIR maps, in Proceedings of the PRIME/URSI Joint Workshop on «Data Validation of Ionospheric Models and Maps», Roquetes (Tarragona) 4-8 May 1992, Memoria, 16, 256-274.

Kecić, Ž.J., P.A. Bradley, Lu.R. CANDER and B. Zolesi (1994): Further test and DGR model ionospheres, special issue on the V Workshop 238 COST PRIME, Annali di Geofisica, 38, 221-232.

LEITINGER, R. (1993): Further proposals for interfacing $f_{0} F_{2}$ and $M(3000) F_{2}$ regional and global maps, in Proceedings PRIME COST 238 Workshop, Graz, May 1993, 91-94.

SINGER, W. and N.I. DVINSKIKH (1991): Comparison of empirical models of ionospheric characterstics developed by means the different mapping models, $A d v$. Space Res., 11 (10), pp. (10) 6.

SINGER, W., J. WeISS and J. BrEMER (1992): Comparison of the improved DGR model, with electron density profiles from VS and TEC data in Europe, in Proceedings of the PRIME/URSI Joint Workshop on «Data Validation of Ionospheric Models and Maps», Roquetes (Tarragona), 4-8 May 1992, Memoria, 16, 60-66.

TITHERIDGE, J.E. (1985): Ionogram analysis with the generalized program POLAN, Report UAG, World Data Center A for STP, NOAA, Boulder, U.S.A.

Zolesi, B., LJ.R. CANDER and G. DE FranCESCHI (1993): Simplified ionospheric regional model, Radio Sci., 28 (4), 603-612.

Zolesi, B., LJ.R. CANDER and P.A. Bradley (1994): Interfacing between regional and global electron density profile, in Proceedings, PRIME COST 238, Eindhoven, 181-194. 